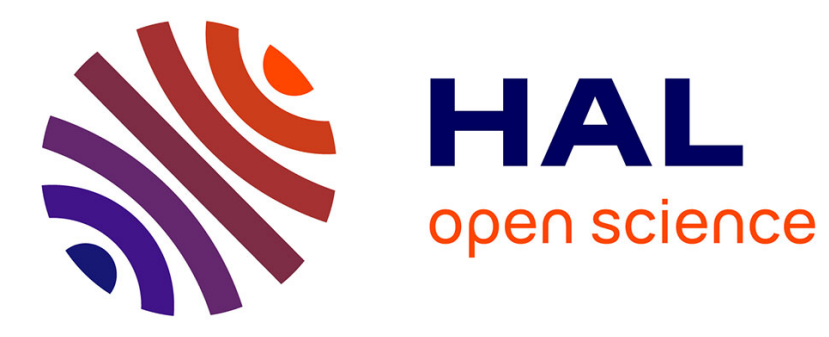

\title{
Generating dance verbs and assisting computer choreography
}

Chi-Min Hsieh, Annie Luciani

\section{To cite this version:}

Chi-Min Hsieh, Annie Luciani. Generating dance verbs and assisting computer choreography. 13th international Conference on Multimedia of the Association for Computing Machinery, 2005, Singapour, Singapore. pp.774-782. hal-00910636

\section{HAL Id: hal-00910636 https://hal.science/hal-00910636}

Submitted on 24 Jun 2014

HAL is a multi-disciplinary open access archive for the deposit and dissemination of scientific research documents, whether they are published or not. The documents may come from teaching and research institutions in France or abroad, or from public or private research centers.
L'archive ouverte pluridisciplinaire $\mathbf{H A L}$, est destinée au dépôt et à la diffusion de documents scientifiques de niveau recherche, publiés ou non, émanant des établissements d'enseignement et de recherche français ou étrangers, des laboratoires publics ou privés. 


\section{Generating Dance Verbs and Assisting Computer Choreography}

\author{
Chi-Min HSIEH \\ ACROE-ICA INPG \\ 46 av. Felix Viallet \\ 38034 Grenoble Cedex \\ FRANCE \\ chimin.hsieh@imag.fr
}

\author{
Annie LUCIANI \\ ACROE-ICA INPG \\ 46 av. Felix Viallet \\ 38034 Grenoble Cedex \\ FRANCE \\ annie.luciani@imag.fr
}

\begin{abstract}
As quoted in the philosophy of contemporary dance: «Understanding the directions for a Free Dance performer stems mainly from the qualities and energy of the movement rather from spatial criteria», a lot of emphasis is put currently on generating computing dance movement by dynamic and energy, which is totally different from producing movement by kinematical based gestures in sequence. We argue that it is an ideal interactive media to connect computer and choreographer.

In this paper, we present a set of dynamic models according to dance verbs: "to rebound", "to jump", "to flip", "to wave", etc, served by physically based particle modeling based on Newton's law. Among them, user has a high-level motion control to modify the quality of such dynamically generated movement, for example, light/strong, free/bound, sudden/sustained, etc. These dynamic models are hence well suited to produce spontaneous motion that looks natural and plausible. To sum up, we propose a methodology, focusing on the birth, the growth and the death of cause, which include mystic anticipation, inner propagation and virtual momentum exchange. Our methodology exhibits energetic succession and connects well the dance, physics and computer. It is convincingly a well-suited direction for computer-aided choreography.
\end{abstract}

\section{Categories and Subject Descriptors}

I.3.5 [Computer Graphics]: Physically based modeling; I.6.8 [Simulation and Modeling]: Types of Simulation: Animation; J.5 [Arts and Humanities]: Performing Arts.

General Terms: Performance, Design.

Keywords: Physically based particle modeling, dance verbs, modular motion design, choreography and dance art.

\section{INTRODUCTION}

Producing human motion by computer animation is significantly analogous to making dances by choreographer. Nevertheless, the

Permission to make digital or hard copies of all or part of this work for personal or classroom use is granted without fee provided that copies are not made or distributed for profit or commercial advantage and that copies bear this notice and the full citation on the first page. To copy otherwise, or republish, to post on servers or to redistribute to lists, requires prior specific permission and/or a fee.

MM'05, November 6-11, 2005, Singapore.

Copyright 2005 ACM 1-59593-044-2/05/0011..\$5.00. interactive degree between choreographer and computer is still far from profound. It infers that the unwillingness of dancers and choreographers to let anything get between them and the live kinesthetic experience [6]. It also reflects that there is some misleading thinking in the developing technology for performance art. Perhaps the computing thinking is not intuitive, not in sufficient accordance with dance thinking.

From an interesting point of view as below, we compare the real scene with the virtual scene created by computer. If we ignore the act of perception in vision, we appreciate illusively two identical scenes. If we put individually an apple into both the real and virtual scenes, we find out immediately and distinctly the difference between the two scenes. For example, the apple in the real scene falls down and stays finally on the floor due to the gravity and the ground's resistance force, while the virtual apple has not this natural movement except if it is designed. In our live world, this kind of interaction, obeying Newton's law, exists all the time although we cannot see it. Similarly but more profoundly, human dancer breathes naturally and this is hypothetically due to the interactions inside our material body, even we cannot see them. Conversely, to produce natural scene in virtual environment, these natural interactions have to be considered.

Here, we boldly propose the difference between generating and looking motions. We suppose that the gap is coming from different cognitions of dancer and audience. Dancer concerns his energy within body and his interaction with environment (gravity, floor, partner, etc) to provoke motion. Audience perceives the resultant motion in visual, spatial and temporal ways; that is to say, the motion is composed of "many gestures". This gap led to two extremes of making motions: computer produces motions from the result; choreographer produces motion from the cause. Therefore, the developed system-communicating computer and dance focusing on kinematical based gestures is generally less suited for assisting choreography.

\section{BACKGROUND}

\subsection{Background}

Badler and Smoliar [1,2] summarized several approaches about digitally human motion representations. In the report of HerbisonEvans [9], he describes various uses of computer graphics in dance: dance notation, choreography, live performance, movement understanding, movement teaching and digital movement representation. 
Based on key-framed interpolation, several applications translate the Laban notation scores [6] or Benesh notation [19] into 3D character. With the help of dance notation, theses works resolve the labor-intensive problem of keyframing. However, dance notation is recorded manually by notation observer, which migh lack the details of interaction with the environment, for example, a little bounce of knee while falling down. For a dancer, this missing can be overcome while for a computer representation, it could have an influence on the movement believability.

Confronted to the difficulty of synthesizing dance motions whether with the symbolic Benesh notation system [3], or with the Laban description of body structure at a specific time $[11,12]$, several approaches focused on adding expressions to the keyframing based motion [7, 21]. Chi et al. [7] proposed the EMOTE model which gives a good combination between key-pose and the Effort-Shape theory of Laban [13] to generate more natural synthetic gestures. Rose et al. [21] proposed methods of multidimensional motion interpolation.

The web3D Dance composer [23] presents dance motions coming from motion capture and connects every motion data based module with help of Laban motif description. Motion data based animation can provide many nuances in the movement, and it is helpful for movement understanding and motion training. However, it is still laborious and costly to modify the data, even though recently some approaches focus on motion editing, transition and synthesizing by algorithm optimization with help of spacetime constraints or kinematics constraints.

For both key-framed and motion data based animation, we should always have a predefined motion, which is less suited for computer-aided choreography. In other word, such method may not be considered to assist a choreographer to design and create dance motion as with the usual high-level metaphoric way: "be as a leaf falling to the floor lightly" or "be as an apple falling to Newton strongly". It cannot focus more on the style of the motion or on the metaphoric conditions to create it than describing explicitly the motion itself.

Instead of this, dynamic approaches are able to generate realistic or plausible motions automatically. There are two main catalogues of dynamic approaches: one is direct dynamic when forces generate the resulting motions directly; the other is inverse dynamic, which means finding the exact forces to generate a desired motion. Recently, several dynamic researches focus on "dynamic controllers" and "spacetime constraints optimization based".

Van de Panne $[24,25]$ used proportional-derivative (PD) controllers that are functionally equivalent to a spring and damper, to determine the internal forces and torques. In this work, a dynamic behavior motion is generated without an explicitly spatial motion trajectory. "Dynamic controller" belongs to "generative models", in which the motion is not explicitly defined in itself but produced by its instinctive forces. That is to say, "dynamic controller" drives the actuator forces based on the current state of the environment. These approaches allow producing various plausible and expressive motions by changing a few of the high-level parameters, they could also exhibit complex evolutions. It enables to take into account the interaction effect with environment. However, "dynamic controller" encounters always the problem of controllability: how to have a desired motion.

Spacetime constraints was proposed by Witkin and Kass [26] to have high motion control by specifying what to do (from here to there) and how to do, without specifying the explicit motion trajectory. Spacetime constraints optimize the resultant motions underlying physically based objective functions, therefore it provides more controllability and realism. However, spacetime constrained optimization based is always time costly and difficult to apply into a complex structure model. Popovic and Witkin [20] drew some ideas from biomechanical literature and proposed a manual simplification to reduce the complexity of structure. Safonova et al. [22] propose the similar idea to reduce character's degrees of freedom automatically. Without doubt, simplifying a complex model reduces the time of computing. Many excellent approaches have proposed algorithmic optimizations to progress this type of problem, but it is still an open problem.

In addition, Gleicher [8] inferred a concept of other possible objective as "gracefully" with meaningful attributes in comparison with energy minimization objective. However, for low-dynamic motion and stylized motion, it is not easy to define suited objective function for optimization. In fact, the main interest of our approach is weather the varieties of expression, for example, a comfortable way to move, an exaggeratedly way to move, or a high dynamic way to move, etc, could be achieved by different objective function

To sum up, the difficulty of dynamic based animation is a delicate problem between motion control, complexity of character structure and computing cost. In this paper, we look backward to "physically based particle modeling" which is a particular kind of "dynamic controller" and has the advantages of an efficiently computing and of a powerful interaction with environment [18]. On account of this, we circumvent the computing problem. The remaining challenge is how to control a desired motion.

To solve this challenge of controllability, we propose the idea of "modular design" according to each kind of movement (i.e. dance verbs). Among motion modules, complex character structure and complete motion are simplified and refined by "the just necessary masses" and "the essential interactions". Thanks to the use of the simplification procedure, we could focus more on the interaction and connection through dance movement and take advantage of the simplest computing technique.

Since this simplification procedure can not easily be defined using simple objective functions, and since we have a wide variety of expression, both stylized motion and low-dynamic motion, we decide to derive them directly according to the dance and physics literature $[4,5,15,16,17]$.

\subsection{Motivation}

In the aim of assisting choreography, the primary principle in our research is exactly the interactive thought between computer and choreographer.

We suppose choreographers are more interested in "cause propagation" and "energy transmission" during every action. Choreographers always abstract dance movements, i.e. a principal action, a reaction, an anticipation, etc, and abstract a simplified character structure as well. Therefore, if we give choreographer an 
animation system having power algorithm to compute the complex dynamic behavior, the choreographer is obliged to think in a completely different way, it is more complex for him.

Thus, our approach, according to choreographer's philosophy, is interested in simplifying dance movements instead of constructing complex dynamic-computing system. In order to refine dance movement, we need to deconstruct character structure into massinteraction as well. In view of dance compositing, we extract dance phrase from dance; we extract dance verbs from dance phrase. Then, we refine a driving cause from every dance verbs. The assisting choreography system we proposed has both the cause and the dance verbs as its origin that is convincingly suitable for choreographer.

\section{SYSTEM OVERVIEW}

Our physically-based particle system, called Cordis-Anima, is a discrete computing algorithm that provides a continuous dynamic system. Cordis-Anima is based on elementary components: inertial type (mass) and interaction type (force). The position of a mass is computed from a force input according to Newton's law. The interaction force is generated according to the position and velocity of the masses. The driving force could be designed by defining the potential energy (initial position and stiffness parameter) or the kinetic energy (initial velocity and mass).

The system is based on two kinds of forces: elasticity and viscosity. Only with elasticity, we are in the case of constructed modeling under energetic consistence that implies an ideal environment. By adding viscosity, we can design a modeling which consumes its initial energy and which presents exactly a real phenomenon in real world. Broadly speaking, derived from elasticity force and viscosity force, there are three principal interaction forces: Elastic-Damping, Collision-Damping and Attraction-Damping. More details are to be found in the paper [18].

Physically based particle models are efficient to simulate the dynamical behavior, in particular for natural phenomenon such as fluid, and deformable solids. However, human motion is a complex biomechanical behavior, rather than a pure behavior obeying law of physics. The originality of our research is that, based on particle modeling, we generate dance movement only with the necessary minimized masses and interaction.

\section{THE METHODOLOGY}

While a complex character structure is deconstructed in a "massinteraction" network, the material skeletons and articulations do not exist anymore. Instead, this reduced number of interacting "masses" represents either materials or functional components. Here, it differs from classical uses of physical modeling to represent exactly the matter properties. Because of this, we are concerned only in the "interaction or propagation" through all the action.

In view of dancing's dynamic, especially for contemporary dance, every action comes from a cause that destroys the initial balance. Then, this cause propagates to trigger the remaining motion. In the end, our body will find another new balance. Consequently, the process of dance has a single common dynamic formula: "a cause trigger-let go and relax- new balance". This pattern is called "preparation-action-recovery".
In our approach, in accordance with the process of dynamic, all the dynamic models are created according to the following procedure: (1) finding out the "motor locus" and initiating the energy into this motor (the birth of cause); (2) generating the motions from the "motor" (the growth of cause); (3) decreasing influence of the motor and finding out another new balance (the death of cause). It is well analogous with, in Chinese calligraphy, a pattern: "drop of stroke - moving brush - closing of stroke".

\subsection{The Birth of Cause}

For every selected dance verb, we will look for the minimum possible and physically consistent plausible cause - called "motor" - that triggers the movement of this verb. This "motor" is the origin of dancing movement. The process to find out such motor for each dance verb is not always easy, neither for a dancer nor for a researcher. It is an implicit feature nested in each performed dance motion that has to be elicited firstly.

According to the "principle of causality" [5], a light transfer of weight is sufficient to bring the steps alive. In other words, the oscillation of the centre of mass involves the steps. Thus, the basin, i.e. the weight center, can be considered as the inner motor for many dance actions. In some other cases, especially for the locomotion, the force coming from the ground through the feet originates the displacement of weight center. That is why the locomotion is triggered only from the physical contact of the feet with the floor. Because of this, the foot-take-off could also be understood as an external and mobile motor.

These two types of motor: one being an "inner motor", the other being an "external motor", can be considered as the two main typical and complementary sources of motion. Once the motor is decided, the subsequent challenge is how to make motor alive, more precisely how to initiate the energy of motor.

Among the twelve Disney studio's principles of animation [14], in some action, the technique "anticipation" is required because of physical reasons, for instance, to squat down is an anticipation to rebound. "Anticipation" helps to guide the audience's eyes to where the action is about to occur; anticipation, including motion holds, is great for "announcing the surprise." To make the analogy with the tradition animation principle, anticipation, we called our process to initiate the primary energy of motor as "mystic anticipation". It stresses the mysterious of anticipation and implies that even we do not understand how our biomechanical body has its proper energy, we still initiate a plausible energy into motor by physics knowledge. Dancer imagines and feels an energy transmission through his/her dancing body, but does not care how the motion is provoked by his muscles and by his biomechanical energy.

In our system, driving motor could be designed by defining the potential energy (initial position and stiffness parameter) or the kinetic energy (initial velocity and mass).

As an example "to rebound", if we add an initial velocity to the center gravity, causing it to fall down, the driving motor thus has a kinetic energy. If we add a potential energy to the squat state, the driving motor would thus have the ability to jump.

Another example is when a dancer twists his trunk like spiral to have torsion before doing a self-turn. During this mystic anticipation, this dancer generates a potential energy in his driving 
motor: gravity center. Therefore, the more torsion is, the more turns there will be. Another situation, a lead dancer does a turn because her leading partner pushes her hand. During this mystic anticipation, the lead dancer has a kinetic energy in her driving motor: hand. The more force pushed into her hand, the more turns she does.

Briefly, once the motors have been chosen and the energy initiated, which is the birth of cause, their force will trigger the motion of the other parts of the body, and will generate the natural movement automatically. That is exactly in accordance with the concept of contemporary free dance.

\subsection{The Growth of Cause}

Applying a triggering procedure from the driving motor, then, we could produce all the evolutions of the positional data, namely the trajectories of movement. The process by which motors provoke the whole motion is as mysterious as beautiful. Dancers exercise their body everyday in the aim to discover and rehearse how the motion of the whole is duly evolved from the motor locus. We identified two basic complementary processes: the "inner propagation" and the "virtual momentum exchange", on which we based all the following models presented in this paper.

Let us imagine a spine moving slowly as fluid wave motion. It exhibits an "inner propagation" from basin to head. "Inner propagation" does not only exist within the real material bones and muscles. A good example is the mimic of soft elastic flying with rigid articulated arms. We can model such "inner propagation" by connecting media: viscous-elastic interaction.

Among the other two animation principles [14], the technique "follow through" consists of the reactions of the character after an action, and it usually lets audiences know how he feels about what has just happened; the technique "overlapping" describes an action taking place because of another action. To expres "followthrough" and "overlapping", we use always "inner propagation" that assists the main action in provoking a reaction or another action. For example, analyzing walking, the step is an action; the balance of arm is a reaction; the cloth and hair are other actions. We could thus connect them by viscous-elastic interaction, e.g. the right foot with the left hand, and vice versa.

Another typical situation is when movement stops suddenly, without any materially apparent reason and restart surprisingly. That is the case when skilled actors mimic the presence of an imaginary wall with their hands. All seems as if there is a perfect momentum exchange between the real body of the dancer and the non-real object, evoked by the mimics. We call this phenomenon, a "virtual momentum exchange" and it can be modeled as a collision during which there is a complete motion quantity exchange from one body (that can be real) to the other (that can be imaginary). This skill of the dancer makes it believable that there exists a perfect momentum exchange between a real body and an imaginary one. We can say "Imaginary physically consistent" action.

To sum up, the approach here is not to model the effective material body, but to design a physically-based model of all what can be the interiorized possible causes of the dance motions as they are felt by the dancer and by the watcher.

\subsection{The Death of Cause}

To admire the beauty of each movement, the moment should be at its end or one must wait till the end.

In this dynamic process, we focus on "recovery" that means to refind a new balance. A driving motor, namely a cause, may remain energetically consistent in the whole dynamic-model; or may be extinguished gradually by the influence of environment, i.e., gravity, friction, etc.

After being influenced by the motor's initial energy and the influence of environment, our body will and should stay at a new balance. With a beautiful ending, the whole effort of every cause is powerful to see and to feel. With a beautiful metaphor, the death of cause is an exhaling breath coming from an inhaling breath and going to another inhaling breath. It is a breathing rhythm for a dancing body and a dancing movement.

Our dynamic system provides an energetic consistence of this environment by elastic interaction and an energy diminution by viscosity interaction. No matter under energetic consistence or energetic diminution situation, our dynamic model always keeps energy succession during all the dynamic process. By the help of viscous damping effort, generating motions have more various nuances.

For example, "to rebound", the different way about energy extinguishing affects different feelings of landing. A strong landing with a full rebounding comes from less energy extinguishing. In contrast, a light landing with some vibration of knee comes from diminishing energy.

\section{SELECTED DYNAMIC MODELS}

In the literature [10], we have proposed some dance verbs to explain the "inner motor" and "external motor" concepts. Some dance verbs as "to sway" "to squat down" "to turn" are classified as "inner motor". Other dance verbs "to walk", "half turns step over step", "swing steps", etc are "external motor" type. In the section 7, appendix, we select the already treated dance verb "to work" [10] to explain the "virtual momentum exchange" concept. In this paper, we add environment influence, e.g. gravity, into the new dynamic models. Farther more, we progress and complete the methodology: the life of the cause. The new results of dynamic models: to rebound, to jump, to flip, to develop and to wave, are presented below and explain our methodology.

\subsection{To Rebound}

Analyzing a simple vertical rebounding, there are some phenomena during this action: The gravity center descends and leads the knee to squat. The knee stretches leads the body to rise up. Finally, the feet and knees bounce and vibrate. By dynamic view, we suppose this action also has 3 periods: 1) The gravity center has a kinetic energy that leads the knee to squat down; 2) The leg has a potential energy, it pushes the floor to compensate the gravity and rebounds; 3 ) The gravity centre has small vibrations and try to find out his new balance. There are exactly three sections according to our methodology: 1) Mystic Anticipation; 2) Inner propagation; 3) New balance (cause frustrating or cause extinguishing). 
In our model "to rebound", such movement is modeled with four masses, one for the basin, one for the foot, one for the knee and one for a virtual-symmetric knee. The virtual-symmetric knee is designed to keep the knee in the right transversal translation direction, because the knee is limited in its rotate degree of freedom in anatomy. Each of them are linked by six viscouselastic interactions (Figure 1).

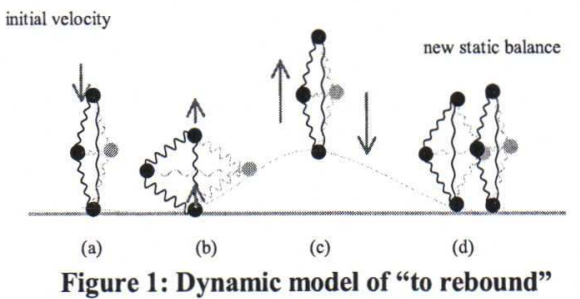

Because the dancer squat down without a conscious thinking of what the explicit gesture of knee should be, we put the weights of mass-knee and mass-virtual knee relatively lighter than the weights of mass-basin and mass-foot. It means exactly that the mass-knee is the reaction to the mass-basin and mass-foot. Because of the physically solid leg, we set the elastic interactions stronger between them (basin and knee, foot and knee, basin and virtual-symmetric knee, foot and virtual-symmetric knee). The viscous-elastic interaction between knee and virtual-symmetric knee decide the vibration of the knee. The viscous-elastic interaction between basin and foot is the main factor deciding the highness of jump and the vertical bounce.

Once the viscous-elastic interactions have been set up, that is to say our simplified character model has the ability to react by a driving force into motor, the user could easily and intuitively control the movement "to rebound" by giving an initial vertical velocity into mass-basin. The higher driving force leads to a deeper squat down and a higher rebound (Figure 2). It reflects well the thinking of dance: to jump higher, dancer needs to squat down more and pushes the floor more. Besides, the user could change the degree of gravity that implies the influence between environment and human action. Thus, this model, "to rebound" can generate different rebounding under the same condition (the same character, the same driving force) in different environments ex, on the earth or on the moon.

As figure 1-(a) shows a driving force with velocity $\left(\mathrm{V}_{0}\right)$ given into mass-basin ( $m$ basin), there exists kinetic energy for the entire model. Then, Figure 1-(b) shows that the entire model stays as dynamic balance without any velocity, so there exist only potential energy. Figure 1-(c) shows the rebounding provoked by potential energy and it finds out another dynamic balance at the highest level.

Through this model, "to rebound", these parameters are well suited for the thinking of dance: the mass is in accordance with body weight; the viscous-elastic interaction is in accordance with our muscle and it is chosen to fit our way to dance. By playing these parameters, we could have a high/low rebounding, rigid/soft landing, sustained/sudden vibration before stopping (arriving to a new balance). Moreover, all the movement is provoked by an initial energy (a driving force given into motor-basin) and all the process is in well accordance with energy transmission, which is treated by "inner propagation" in our approach. It is thus an ideal interactive way to assist choreography and includes the philosophy of dance dynamics into science technology.

(a)

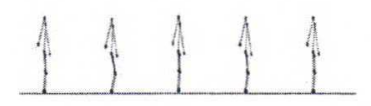

(b)

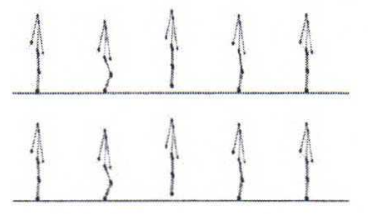

(d)

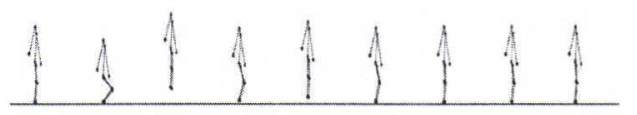

Figure 2: (a) shows that an initial force $V_{0}=15$ leads a little rebounding; (b) shows $V_{0}=30$ leads a higher rebounding; (c) shows $V_{0=}=45$ has the highest rebounding; (d) shows $V_{0}=45$ with more vibration of knee.

\subsection{To Jump}

Jump is a so beautiful movement that expresses the dream of flying and the courage to resist to our physical world. Without doubt, jump is a very important action, and that dancers have interpreted many varieties of jump by composing with two legs' take-off, flying and landing. In the table 1, we list five basic forms of jumps: Jump from both feet to both feet; Leap from one foot to the other foot; Hop one foot to the same foot; Assemble from one foot to both feet; Sissonne from both feet to one foot.

Jump consists of a horizontal motion such as the "grand jete" and a vertical motion such as "a free falling object". When the body is thrown in the air, the gravity center's trajectory is a parabolic curve. In physics, the parabolic trajectory of a projectile is decided by: the initial velocity composed of horizontal and vertical components, the weight and the gravity. Kinetically, the projectile motion could be seen as the abstraction of a jump movement while flying.

Farther more, in view of dynamics, for a jump action, both takeoff feet receive their momentum because of pushing together the ground. For leap action, the take-off foot receives its momentum because the other foot squat down and pushes the ground. In our live experience, to leap from one leg to the other leg, we always care about the support-leg pushing the floor to provoke the other leg to take off. However, what happened indeed between the support leg and take-off leg is complex and the complete mechanism could not be virtually implemented. In fact, human body is a self-driving motor so complex in biomechanics that we do not know how human anticipate the exact quantity of energy necessary to act. In our approach, it is exactly "mystic anticipation". 
Table 1: Five types of "to jump"

\begin{tabular}{|l|l|l|}
\hline & Form & Modeling \\
\hline Jump & $\begin{array}{l}\text { from both feet to } \\
\text { both feet }\end{array}$ & $\begin{array}{l}\text { two identity strong legs; two } \\
\text { take off driving force }\end{array}$ \\
\hline Leap & $\begin{array}{l}\text { from one foot to } \\
\text { the other foot }\end{array}$ & $\begin{array}{l}\text { two identity strong legs; one } \\
\text { take off driving force }\end{array}$ \\
\hline Assemblé & $\begin{array}{l}\text { from one foot to } \\
\text { both feet }\end{array}$ & $\begin{array}{l}\text { two identity strong legs; one } \\
\text { take off driving force; } \\
\text { quickly inner propagation }\end{array}$ \\
\hline Hop & $\begin{array}{l}\text { one foot to the } \\
\text { same foot }\end{array}$ & $\begin{array}{l}\text { two different strong legs; } \\
\text { one take off driving force }\end{array}$ \\
\hline Sissonne & $\begin{array}{l}\text { from both feet to } \\
\text { one foot }\end{array}$ & $\begin{array}{l}\text { two different strong legs; } \\
\text { two take off driving force }\end{array}$ \\
\hline
\end{tabular}

In this "mystic anticipation", instead of looking for how the takeoff foot has its momentum, we are looking on how its momentum acts physically on the remaining action. We simplify the problem of getting the primary energy with an intuitive and plausible designing (motor initiating). So, the take-off foot is initiated with a kinetic energy that implies an explosion immediately of this fly leg. Then the fly leg will trigger the support leg to fly. In the same way, jump action occurs on both take-off feet, we thus initiate kinetic energy into both feet. Briefly, our approach focuses on plausible dynamic anticipation instead of gesture anticipation to jump; this "mystic anticipation" generates jump action physically and variously.

To model the series of "to jump" action, we derive each leg from the prototype of "to rebound" model. We connect the right and left legs with a viscous-elastic interaction between both massbasins. In addition, we can also connect both mass-feet to achieve certain various jumps. Thus, the energy could be transmitted between two legs due to "inner propagation" (Figure 3). Figure 3(a) shows "mystic anticipation" of dynamic model "to leap". In the first step of "mystic anticipation", this dynamic model is under "squat down" state, which means it has potential energy to rebound vertically. In the second step of "mystic anticipation", we initiate kinetic energy to provoke a horizontal locomotion by two forward horizontal driving forces. One is given into basin and leads to the advancement of the whole body; another is given into take-off foot and leads to a "fly forward" movement for the takeoff foot. Then, figure 3-(b) shows the "inner propagation" during which the advance momentum is transferred from take-off leg to support leg with the help of basin's viscous-elastic interaction. In the end, figure 3-(c) shows the dynamic model, which finds out its new balance under the environment influence.

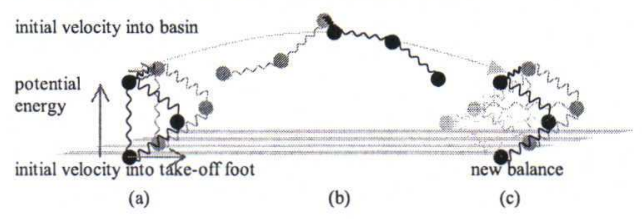

Figure 3: Dynamic model of "to leap"

As mentioned, by initiating a kinetic energy into one foot or two feet, we decide to take off with one foot (leap) or two feet (jump) and also the distance of flying. By designing the parameters of legs' viscous-elastic interactions between basin and foot, two legs will have different strong muscles, which means that the two legs have different potential energy. Thus, it leads the difference about fly time and highness of jump (hop and sissonne). In case of "assemblé", it acts in the same way: to take off with "leap"; then, the interaction between two legs (inner propagation) decides the transfer of advance momentum, so it decides if two legs land together (assemblé) or not (leap).

To sum up, all the varieties of jump action are based on a typical action opposing the gravity. We have provided the powerful way of generating such varieties of jumps just by initiating "mystic anticipation". Therefore, we believe that dynamic generation is more intuitive and physically plausible than kinematics based animation.

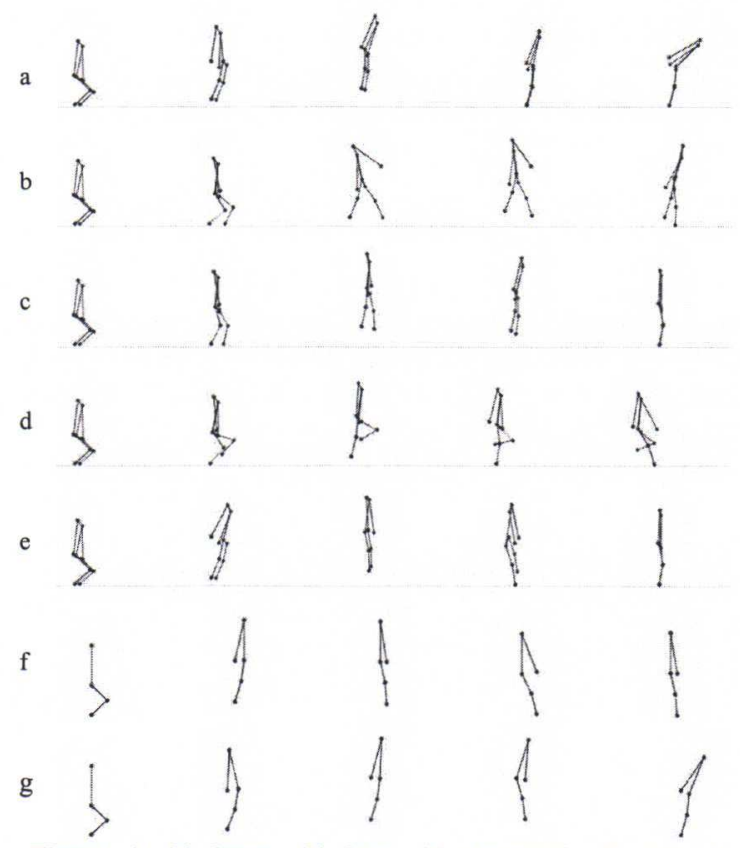

Figure 4: (a) jump, (b) leap, (c) assemblé, (d) hop, (e) sissonne, (f) a bound jump, (g) a free jump.

\subsection{To Flip}

Flip is a jump action combined with an added self-rotation (Figure 5). Derived from "to jump", we could have another dance verb "to flip" by initiating "mystic anticipation" differently. In the "mystic anticipation", we design a horizontal velocity $\mathrm{V}_{1}$ into take-off foot, and another horizontal velocity $V_{2}$ into take-off basin.

Because mass-knee is relatively lighter than mass-basin and massfoot, we simplify the leg model as Figure 6. By playing these parameters, the difference of velocity $\left(V_{1}-V_{2}\right)$ decides the forward or backward direction. Using the difference of angular velocity $\left(\omega_{1}-\omega_{2}\right)$ expression, one decides the direction of rotation. Figure 7-(a) shows backward flip to back; (b) shows backward flip stay; (c) shows forward flip stay; (d) shows forward advance. We illustrate, thus, a simplification of one leg's flip. In a similar way with "to jump by two legs or one leg", we can derive wide varieties of flip action. 
To sum up, this "to flip" model presents the powerful way of "mystic anticipation" that allows to obtain such wonderful results only by modifying some parameters. It means a high-level and intuitive motion control. Besides, from "rebound" through "variety of jump" to "variety of flip", one has the possibility to develop the existing dynamic models and to combine them into a more complex model. It shows the advantage of modular motion design by physically based particle modeling. Moreover, even though the "to flip" is a highly dynamic and complex motion in the whole character structure, in the frame of dynamic model, "to flip" is refined as two principal masses with one principal interaction (Figure 6). Thus, the velocity of rotation can be designed easily by this simplest pattern: (drivingV $\mathrm{V}_{1}$ and driving $\mathrm{V}_{2}$ ). However, the most important thing in this model is how to perform the rotation movements rather than the velocity value.

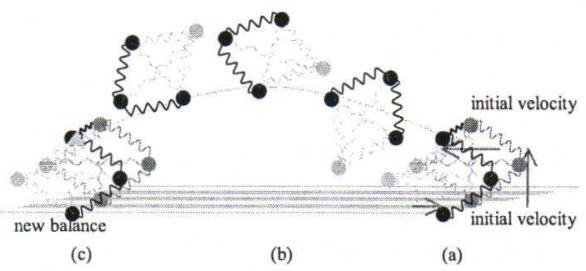

Figure 5: Dynamic model "to flip backward"

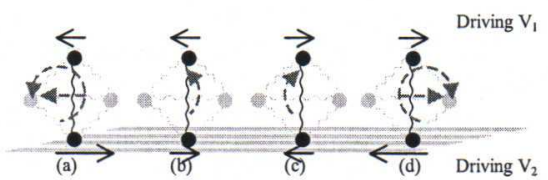

Figure 6: The simplest pattern of "to flip"

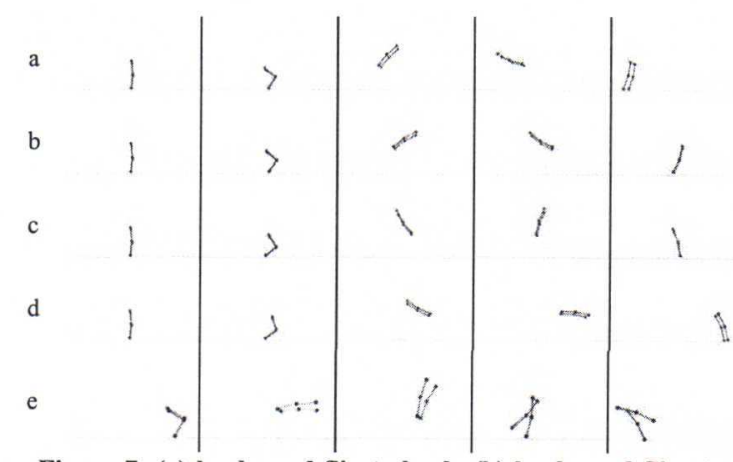

Figure 7: (a) backward flip to back, (b) backward flip stay, (c) forward flip stay, (d) forward flip advance, (e) a variety of backward flip.

\subsection{To Develop and To Wave}

The "to develop" movement is a very slow and continuous movement of unfolding an arm or a leg, resulting from pulling the elbow, which in turn induces the motion of the others arm segments (wrist, hand, fingers). The motion along the arm is like a propagation of a sea wave from the elbow to the fingers. We modeled this motion with four masses, respectively for the shoulder, the elbow, the hand and the finger, linked with simple visco-elastic interactions. The visco-elastic interaction force can produce a jerky/bounded movement or a soft/fluid movement, according to the values of masses $(\mathrm{m})$ and of visco-elasticity $(\mathrm{k}, \mathrm{z})$ between the elbow, the hand, and the fingers. The driving force could be an external motor, which impact on the mass-elbow for provoking each wave propagation. While this dynamic model "to develop" connects with other dynamic model hierarchically, a driven mass from other dynamic model may be designed as the driving motor into "to develop".

Spine is the spring (origin) of all the movement from biomechanics point of view. Here we propose a dynamic model "to ripple" that has an expression of wave propagation under material spine. When the dynamic model "to ripple" stays in the static balance, it does a breathing motion as a real human. After an external driving force sets the spine into action, an influence propagates from spine until the end of limbs. Through this dynamic model, we obtain a sustained and continuing movement with breathing. It is so naturally soft in comparison with a mechanical movement from a robot. This kind of movement is a suited presentation for contemporary dance.

Dolphin is a similar movement to ripple. The difference is that the dolphin motion starts wave motion from a head; ripple motion starts wave motion from the basin. From this model "to ripple", by modifying the parameters, several varieties of wave motion, i.e. swimming, could be obtained.

In brief, "to develop", "to ripple", and "to dolphin" have the common pattern: wave propagation, this pattern could be simulated easily and suitably by our methodology "inner propagation". Furthermore, because of hierarchical modularity propriety, it is possible to compose these dance verbs, for example, wave propagation from basin to fingers. It shows not only the possibility of composition but also the property of energy succession under the same rhythm and the same original motor.

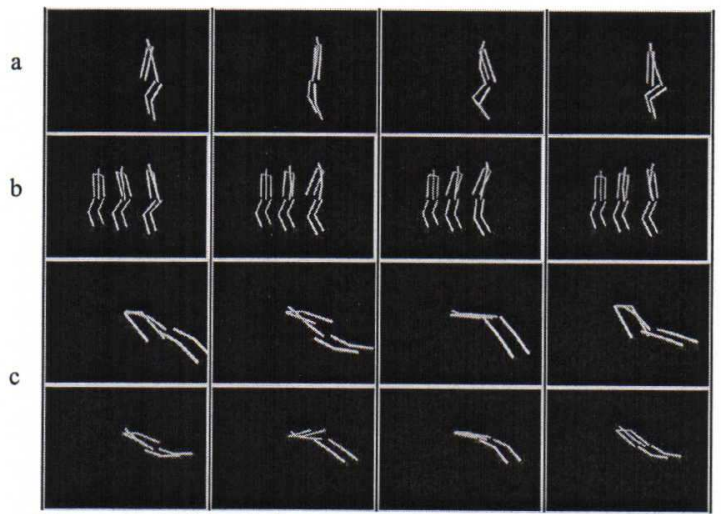

Figure 8: (a)"ripple" movement; (b) the different degree of driving force; (c) a variety of wave movement: swimming 
(a)<smiles>CCCI</smiles>

(b)

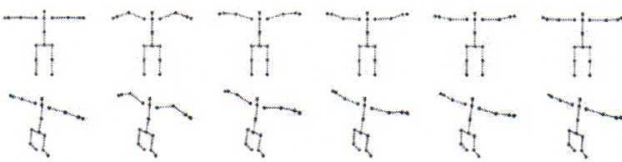

(d)

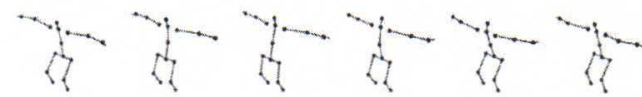

Figure 9: (a) "ripple" movement; (b) "arm wave movement"; (c) "arm wave movement" is added to "ripple movement" without interaction between them; (d) "spine wave movement" connects with "arm wave movement" dynamically, that is to say there exists interaction between them.

\section{CONCLUSION}

As mentioned before, the different way to implement computerchoreographer communication is the way to produce desired motions, one from the result; one from the cause. We indicate another interesting view starting with the question does human consciously think of how much force he should have to perform a desired motion? Computer is passive and needs to be told the exact forces or the rule to obey (i.e. constrained optimization). Conversely, human is sensible to the force change, and concerns always himself with all the interactions. In our research, we argue the instinctively different capabilities of computer and human. We hypothesize dancer has a good sensibility to feel the interactions and to be influenced by these interactions. Because of this, we constructed an interacting network instead of algorithmic progressing.

In this paper, to find out the minimum but sufficient number of interactions, we deconstructed character structure as "massinteraction" network keeping always the essential interactions; and we extracted dance verbs to be used for modular motion design. Then, we proposed the methodology for energy processing of every dance verbs, the life of the cause. All mentioned above aims to bridge the gap between computer and choreographer and to assist choreographer, computer has to produces motions from the cause not from the results, and has to consider the interactions inside the body and with environment.

The proposed methodology is well adapted to the philosophy of contemporary dance, in which the movements of body parts are not explicitly dirigible in a prescriptive way but provoked by the dynamic status. In these dynamic models, they provide not only an intuitively high-level motion control, but more important, it is possible to modify the quality of movement such as light/strong, free/bound, sudden/sustained. Without doubt, to produce digital dance motion and have its own style and quality as well, is a wonderful aim in our research.

Briefly, these physically-based particle models lead to define physically-based metaphors as they are lived by the dancer and used by the dance teachers: motors of motion, propagation, external resistance, full momentum transfers, etc. It leads to a type of minimal model, producing the quality of the motion in a "natural way of performance and thinking". In addition, these models allow to be combined in order to produce more complex dance motions. The future direction would be to constitute library of dance verbs models, and to progress composition methods of these models. More profoundly, the aims in our methodology could focus on bringing a death of cause into the birth of another cause.

\section{APPENDIX}

Here we choose a typical locomotion: walk, to explain the "virtual momentum exchange" concept in our methodology. In the direction of the displacement, when the foot stops, all it happens like the foot looses its momentum in a similar way when it hits an imaginary medium and this medium returns the momentum back to the foot after a certain time (exactly after the period of the step) letting the foot to be propelled. Such process is what we called, "virtual momentum exchange", able to stop the motion and to restart it under an energy conservation condition.

The below figure shows a walking form right side to left side. Each snapshot presents "virtual momentum exchange" between the right foot (a red sphere) and the virtual mass (the circle) or between the left foot (another red sphere) and the virtual mass.

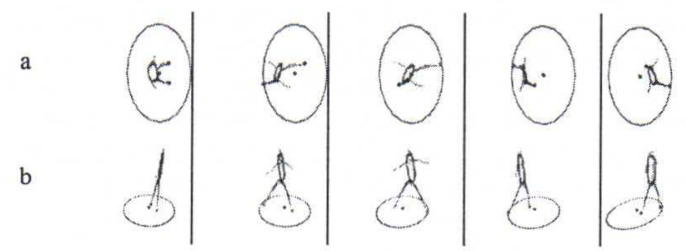

In our "mass-interaction" network, the methodology to coat (the visualization) the motion is another stage of modeling. This coating may be simple, as in motion-capture philosophy. It could also be understood in a more general way as clothing of a pointbased skeleton. There is a wide variety of visualization. Here, we list some results with visual effect.
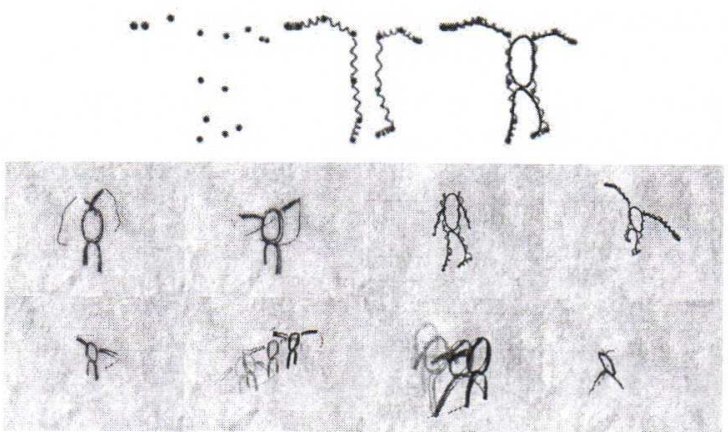

\section{REFERENCES}

[1] Badler N.I., Smoliar S.W.: Digital Representation of Human Movement. Computing Surveys, Vol. 11, No. 1, page 19-38, March 1979.

[2] Badler N.: A computational alternative to effort notation. In Gray, J.A., editor, Dance Technology: Current Applications and Future Trends. National Dance Association, VA, 1989. 
[3] Benesh R., Benesh J.: Reading Dance: The Birth of Choreography. McGraw-Hill Book Company Ltd, first edition 1983.

[4] Bishko L.: Relationships between Laban Movement Analysis and computer animation. In Dance and Technology I: Moving Toward the Future, pp. 1-9, 1992.

[5] Bodak Suzanne : Mémoire vive d'un héritage la danse libre de François Malkovsky. ISBN 2-9516516-0-0, 2001.

[6] Calvert T., Wilke L., Ryman R., Fox I.: Applications of computers to Dance. IEEE computer Graphics and Applications, Vol 25, No 2, pp 6-12, March-April, 2005

[7] Chi D., Costa M., Zhao L., Badler N.: The EMOTE Mode for Effort and Shape. In Proceedings of SIGGRAPH'00, pp.173-182, July 2000.

[8] Gleicher M.: Motion Editing with Spacetime Constraints. 1997.

[9] Herbison-Evans D.: Dancer and Computer: A Potential for Graphic Synergy. Basser Department of Computer Science, Technical Report 422, University of Sydney, 1991.

[10] HSIEH C.-M., Luciani A.: Physically-Based Particle Modeling for Dance Verbs. In Proc. of Graphicon'05, page 85-92, Novosibirsk, Russia, 2005.

[11] Laban R.: Choreutics. MacDonald and Evans Ltd, second edition, 1966.

[12] Laban R.: The Mastery of Movement. Plays, Inc., Boston, 1971.

[13] Laban R., Lawrence F.C.: Effort: Economy in Body Movement. Plays, Inc., Boston, 1974.

[14] Lasseter J.: Principles of traditional animation applied to 3D computer animation. In Proc. of SIGGRAPH'87, volume 21, pp. 35-44, July 1987

[15] Laws K.L.: The Physics of Dance, Schirmer Books (New York), 1984.

[16] Laws K.L., Harvey C.: Physics, Dance, and the Pas de Deux, Schirmer Books (New York), 1994.

[17] Laws, K.L.: Physics and the Art of Dance: Understanding Movement, Oxford University Press (New York), 2002.

[18] Luciani A., Jimenez S., Florens J.-L., Cadoz C., Raoult O. Computational physics: a modeler simulator for animated physical objects. Proc. of Eurographics'91, Vienna, Austria, September 1991.
[19] Neagle R., Ng K., Ruddle R. A.: Developing a Virtual Ballet Dancer to Visualize Choreography. In Proceedings of the AISB 2004 on Language, Speech \& Gesture for Expressive Characters (AISB'04), page 86-97, 2004.

[20] Popovic Z., Witkin A.: Physically Based Motion Transformation. In Proc. of SIGGRAPH'99, volume 33, page 11-20, 1999.

[21] Rose C., Cohen M.F., Bodenheimer B.: Verbs and adverbs: Multidimensional motion interpolation. IEEE Computer Graphics \& Applications, 18(5), September-October 1998.

[22] Safonova A., Hodgins J.K., Pollard N.S.: Synthesizing Physically Realistic Human Motion in Low-Dimensional, Behavior-Specific Spaces. In Proc. of SIGGRAPH'04, August 2004.

[23] Umino B., Longstaff J. S., Soga A.: Laban Motif of Algorithms for Automatic Generation of Dance Sequences in "Web3D Dance Composer". In 24 $4^{\text {th }}$ Biennial Conference of ICKL, London, August 2005.

[24] van de Panne M., Fiume E.: Sensor-actuator networks. In Proceedings of SIGGRAPH'93, pp.335-342, August 1993.

[25] van de Panne M., Kim R., Fiume E.: Virtual wind-up toys for animation. In Graphics Interface '94, pp.208-215, May 1994.

[26] Witkin A., Kass M.: Spacetime Constraints. In Proc. of SIGGRAPH'88, volume 22(4), page 159-168, 1988.

\section{ACKOWLEDGEMENTS}

This work has been supported by the French Ministry of Culture and by the FP6 European Project "Enactive Interfaces" IST 2002002114.

\section{About the authors}

Chi-Min HSIEH is a Ph.D. student at the lab. ICA-ACROE at Institut National Polytechnique de Grenoble FRANCE. He received his Master Fine Art at Taiwan and his Master Computer Graphics in France. $\mathrm{He}$ is doing his $\mathrm{PhD}$ in Computer Choreography.

Annie LUCIANI received the degree in electrical engineering (ENSERG 1973) and the PhD degree in computer science (1984) from the INPG in Grenoble, France. She is a research engineer of the French Ministry of Culture. She is in charge of the ICAACROE research group supported by the French Ministry of Culture and by the INPG. 